\title{
Covid-19, and Hip Fracture Mortality Rates in the Elderly at One Year: Impact and Implications
}

\author{
Ray Marks
}

Department of Health and Behavior Studies, Columbia University, Teachers College, New York, NY 10027, USA

*Corresponding Author: Dr. Ray Marks, Department of Health and Behavior Studies, Teachers College, Columbia University, Box 114, 525W, 120th Street, New York, NY 10027, United States.

\begin{abstract}
Both the presence of the corona virus (COVID-19), plus the widespread prevalence of hip fractures, are immense current major health concerns in most aging nations. This review examines whether there is any tangible impact on survival rates among older adults admitted to hospital in the past year (January 1,2020-March 1,2021) in the midst of the persistent COVID-19 pandemic. It specifically explores if more dedicated and focused preventive efforts are warranted in this regard, if indeed, a third wave of this infectious disease is on the horizon, and vaccines may not prove 100 percent effective, as is predicted by some. To address these questions, this present review examines pertinent literature sources published in the peer reviewed English language between January 12020 and March 1, 2021 concerning hip fractures relative to COVID-19 regardless of report type. The aim was to identify if specific interventions to offset the risk of incurring both COVID-19, as well as hip fractures are warranted at this time of widespread lock downs and service closures or delays. In particular, support for an enhanced focus on preventing both COVID-19 infections as well as hip fracture injuries was examined. Based on available data located in PUBMED and other electronic data bases, we conclude older adults who sustain a hip fracture, especially the modest percentage testing positive for COVID-19 are potentially at a higher risk for premature mortality than comparable cases with no infection. To avert any preventable excess suffering along with the immense social costs experienced by the both the affected individual, as well as society, heightened efforts to minimize COVID-19 infection risk, along with standard falls prevention approaches appear imperative especially among isolated older adults suffering from osteoporosis, poor nutrition, pain, cognitive challenges, and muscle weakness.
\end{abstract}

Keywords: COVID-19, falls, frailty, infection, hip fractures, intervention, morbidity, mortality, older adults, prevention, surgery.

\section{INTRODUCTION}

In recent years, hip fractures have been viewed as one of the most costly and serious health care problems facing policy makers, health care providers, and organizations in the context of aging societies. Indeed, despite some evidence of declining hip fracture prevalence rate in recent decades [e.g., 1, 2], the injury remains an ever present and potentially unwarranted cause of severe disability, excess morbidity, reduced life quality and premature mortality among older adults [3]. Moreover, according to some, it is just as likely that the annual incidence of hip fractures could increase, rather than decrease, over the next several decades [4], with fragility hip fractures having an estimated expected prevalence rate of 6.3 million cases world wide by 2050 [5]. In addition, data do not usually account for the fact that a 'first' hip fracture incident may increase the risk of a 'second' similar injury, either on the same side, or the opposite hip, plus other non-hip fragility fractures that require hospitalization. Data do not account furthermore for other possible associated hip fracture-related complications [3], plus a susceptibility to frailty, along with heightened COVID-19 viral infections, that have produced many fatalities among the older population, since the onset of the corona virus pandemic that began in Wuhan, China in early 2020. Moreover, older 
Covid-19, and Hip Fracture Mortality Rates in the Elderly at One Year: Impact and Implications

individuals, living either in the community or an institution already infected by COVID-19 that is only confirmed when they are admitted to hospital with a hip fracture are likely to be negatively impacted as implied by research by Wang et al. [6].

Thus, in both cases, currently admitted older adults who have sustained a hip fracture may not only be expected to be at a higher risk for excess morbidity than in pre-pandemic times for comparable injuries, but to possibly be at an increased risk for premature mortality.

Given that the prevailing overall burden, as well as the projected public health care burden of this debilitating injury that is not limited solely to the costs of functional disability and increased death rates, but commonly also to a loss of the independent functional ability of the injured adult, plus related anaesthesia, nursing care, long-term care and surgery costs [7-9], are more intense preventive strategies against hip fracture as outlined by Wilson and Wallace [10] desirable in this regard and in the current persistent pandemic period?

This question, is not only academic in our view, but clearly has important economic and social implications, in a period where any persistent social isolation measure leading to reduced self-care ability, health access, plus possible heightened emotional distress, depression and fears that may heighten the overall risk for both fragility as well as falls, sleep and poor nutrition may prevail.

Accordingly, this present review aimed to examine the extent of support for the idea that more intense preventive strategies against hip fracture injuries, as well as COVID-19 among the at risk elderly living in the community are warranted.

To this end, we specifically elected to examine whether there is a steady incidence of hip fracture injuries that are possibly requiring intense care in the hospital, plus whether there are higher death rates among those who test positive for COVID-19. Since COVID-19 is prevalent worldwide, and most societies are aging, and may be severely challenged at this pandemic time, we aimed to examine and describe the early mortality rates and other data pertaining to hip fracture samples that have been examined to date during the coronavirus pandemic to provide clues about any possible preventive factors that may have been overlooked. A second aim building on this was to derive any lessons that could be applied currently at minimal cost, so as to possibly minimize hip fracture injuries in the event of a 'third wave' of the virus.

\section{RATiONALE}

The frail elderly who are at high risk for hip fractures, as well as hip fracture survivors and those with one or more common co occurring chronic health conditions, such as diabetes, are an important subgroup of older adults who do not appear to have been targeted intentionally or systematically in the context of community based settings during the present pandemic. This is unfortunate because evidence suggests, social isolation, limited home care services, muscle weakness, fatigue [11], and cognitive fear factors and others put forth to prevent the risk of COVID-19 exposure, are also those actions that can readily heighten frailty, fragility, and falls risk.

Unsurprisingly, Bhat etal. [12] found falls that occurred domestically to increase rather than decrease during a recent lockdown period, as did hip fracture injuries among the elderly over a similar period of lockdown in Italy [13]. However, even if this is only due to trauma experienced by unexpected slips, trips, or falls from a height due to the adoption of new modes of movement, or the need to carry out movements that are risky [11, 14], health directives to avert this major cause of hip fractures may have been eliminated or impossible to access. Along with possible cuts to health services and resources, it is also plausible in our view that due to their increased vulnerability for falling, as well as COVID-19 infections, a fair proportion of older community dwelling older at risk adults who were not prepared for any sudden withdrawal of health promoting services [15], would tend to continue to incur falls and bone fractures, including more complicated hip fracture injuries, and possible delays in surgery if they have COVID-19 infections l [16].

They may also recover more slowly from both hip fracture surgery as well as any prevailing COVID-19 disease when compared to healthy older adults, for example if vitamin D/calcium supplements are not forthcoming as indicated [15]. They may also become more vulnerable to re infections, as well as future falls and fractures, even if timely surgery and rehabilitation strategies for restoring functional recovery post-hip fracture surgery are available. 
Covid-19, and Hip Fracture Mortality Rates in the Elderly at One Year: Impact and Implications

As per Narang et al. [17] since non-operative treatment is not appropriate due to an even higher mortality rate, it was deemed important to examine mortality rates among the surgical hip fracture candidates independently to ascertain if COVID-19 exacerbates the outlook for the current hip fracture surgical candidate. We elected to focus predominantly on the home as the key setting where many hip fractures tend to currently occur [18], and where home care and rehabilitation services are likely to be limited for some time to come.

\section{Method AND Procedures}

To fulfil the aims of the present report, all pertinent full length published studies in the English language detailing the impact of COVID-19 on hip fractures in the PUBMED data base over the time periods January 1 2020-March 1 2021, using the key words 'COVID-19 and hip fractures |', 'COVID-19 and fractures' were sought and examined. Also reported are some findings regarding prior hip fracture prevention recommendations in addition to background information. The focus however was on outcome studies, or studies that described short term mortality rates post hip fracture hospitalization during COVID19. Those search engine data discussing changes to hospital procedures or recommended changes in the future rather than the hip fracture outcomes of surgically treated emergency medicine hip fracture patients, as well as papers under review were not included. To provide a comprehensive overview of the research findings, a descriptive and narrative analysis and summary was pursued.

\section{RESULTS}

Among the available 90 English language studies published between January 1, 2020 and March 1, 2021, most were retrospective reports stemming from China, Italy, France, Poland, Portugal, Scotland, the United States, the United Kingdom, Spain, and Turkey covering the outcomes such as 30-day mortality rates over the time periods March 2020-May 2020. Among these heterogeneous reports, as outlined in an early report [19], it appears safe to say that regardless of data source or venue, hip fracture patients admitted to hospital over the past year are clearly a particularly vulnerable population of older adults, and a group who may experience a higher 30-day post surgical mortality rate than the norm, especially if they are COVID-19 positive. For example, even if COVID-19 had no impact on three hip fracture cases reported in one study [20] and in a 30-day comparative study [21], researchers in Spain found that of the 136 admitted cases they surveyed and where the total mortality rate was 9.6\%, 23/62 cases tested positive for COVID 19, and among these cases there was a $30.4 \%$ mortality rate at a mean follow-up time of 14 days, compared to $10.3 \%$ for patients with a negative COVID-19 test result [22]. This heightened mortality rate among those testing positive for COVID-19 disease was not unlike that reported by Biarnés-Suñé et al. [23] among 68 admitted hip fracture cases, of whom 18 were COVID positive, and where recent in-hospital mortality rates for operated patients was $20 \%$ in those with a positive test, compared with $2.3 \%$ in the group of patients who tested negative. After 30 days, the mortality rate was $40 \%$ in the group with positive tests compared to $6.8 \%$ in the non infected patients. The finding also concurred with a report by Hall et al. [24] in a United Kingdom study where 30 day mortality rates in hip fracture cases with a positive test when adjusting for age, sex, residence type were significantly higher for those hip fracture cases positive for COVID-19.

Arafa etal. [25] too who collected data in a retrospective study of 157 hip fracture cases admitted in the United Kingdom between May 2019 and 2020 and where the 2020 patients were grouped as being COVID positive or negative found hip fractures and inpatient falls to increase by $61.7 \%$ and $7.2 \%$ respectively in the 2020 group. The mortality rate in the COVID positive group was $36.8 \%$ compared to $11.5 \%$ in 2019 , a rate not unlike the $41.2 \%$ mortality rate observed by De et al. [26] for COVID positive hip fracture cases. Hospital stay was longer in that group as well. Similarly Ward et al. [27] in conducting a case control study of 132 hip fracture patients, $34.8 \%$ of whom had a positive COVID-19 tests had higher 30-day mortality rates of $37 \%$ when compared to those with no infection with a rate of $10.5 \%$.

In an analogous study by Egol et al. [28] where $12.2 \%$ of the hip fracture cases were found to be affected by COVID-19, those with a confirmed COVID diagnosis had an increased mortality rate, a lengthier hospital stay, a greater rate of major complications, and postoperative ventilator needs. The morbidity as well as mortality rates were clearly higher in cases with confirmed COVID-19 infections, a finding supported by Kayani et al. [29] in a multicenter cohort study of patients being treated surgically in Greater London 
Covid-19, and Hip Fracture Mortality Rates in the Elderly at One Year: Impact and Implications

across nine hospital sites where COVID-19 positive cases had three times the rate of postoperative mortality than negative cases. There were also more post surgical complications in the infected hip fracture cases, plus an increased length of stay.

Work by Al Farii et al. [33] has further confirmed that elderly COVID-19 infected hip fractures cases do appear to have a higher 30-day mortality rate compared to uninfected cases, as discussed by Kumar et al. [31] and corroborated by LLevot et al. [20], Crozier-Shaw et al. [32], Lebrun et al. [33], even though Cheung and Forsh [34] who conducted a retrospective study of 10 patients $\geq 60$ years of age with a hip fracture and COVID-19 who underwent surgical treatment in New York City during the COVID-19 outbreak from March 1, 2020 to May 22, 2020 concluded that hip fracture patients who present with asymptomatic or mild COVID-19 infection can safely undergo early surgical intervention after appropriate medical optimization. However, as outlined in Table 1, in addition to studies cited above, a sizeable number of further studies do not echo this aforementioned view. Thrakar et al. for example [35] who examined 43 hip fracture cases admitted in 2020 in a United Kingdom setting, found a 30 -day mortality rate of those $26 \%$ of hip fracture cases with a concomitant COVID-19 infection diagnosis to be $16.3 \%$, which was higher than that of the observed control non-infected groups. Fadulelmola et al. [5] who similarly investigated the effect of COVID-19 infection on 30-day hip fracture mortality among 75 adult hip fractures over the periods March and April 2020 and where the COVID-19 infection rate was $26.7 \%$, also found a significant difference in the 30-day mortality rate in COVID-19 positive group, which was 50\% compared to COVID-19 negative group, which was 7.3\%.
Moreover, consistent with other authors examining similar data sources, Narang et al. [17] who conducted a multicentre prospective study across ten sites in the United Kingdom as regards actual and expected 30-day mortality rates found this to be significantly higher than expected for 2020 COVID-19 positive patients, with 30 observed deaths compared against the 10 expected from prior hospital based risk stratification data. This was also the finding of Rasidovic et al. [36] who strove to describe the risk of mortality among patients with a fractured neck of femur in England during the early stages of the COVID-19 pandemic, using a multicentre cohort study approach across ten hospitals from 1 March 2020 to 6 April 2020, and patients $\geq 60$ years of age admitted with hip fracture and a minimum follow-up of 30 days. Unsurprisingly, Wang et al. [37] who found a relative risk for postoperative mortality in COVID-positive hip fracture patients compared to non-COVID patients to be 5.66 .

In this aforementioned study, as observed in a recent 2021 review of 580 hip fracture cases, designed to compare pre COVID patient profiles to COVID-19 profiles, mortality rates were likewise increased [38]. Lim et al. [39] as well as Clement et al. [40] also report hip fracture cases with a dual COVID-19 diagnosis have a significantly lower survival rate when compared with those without a proven infection, even though this was not the finding of Serra-Torres et al. [41]. This seven-fold increase in risk of mortality in patients with hip fracture identified by Lim et al. [39] is not likely to abate any time soon, as rates of hospitalization during the pandemic have also tended to increase in some cases in their own right, rather than decrease [42], and hip fracture cases are also likely to develop COVID-19 postoperatively [40].

Table 1. Summary of Key Studies Showing 30-day Mortality Implications for Hip Fracture Surgery COVID-19 Positive Patients 2020-2021 Predominantly During The First Wave as Drawn from the PUBMED Database

\begin{tabular}{|l|l|l|}
\hline Group & Approach & $\begin{array}{l}\text { COVID-19 Associated Hip Fracture } \\
\text { Surgery Mortality Rates }\end{array}$ \\
\hline & & \\
\hline Al Farii et al. [30] & Meta analysis- 4 articles + case series & Increased \\
\hline Biarnés-Suñé et al. [23] & Retrospective study-63 patients & Increased \\
\hline Burahee et al. [44] & Retrospective cohort study- 29 cases & Did not increase* \\
\hline Arafa et al. [25] & Retrospective study- 157 patients & Increased \\
\hline Crozier-Shaw et al. [32] & Retrospective study- 36cases/45 controls & Increased \\
\hline Clement et al. [40] & Multi center retrospective study-1569 cases & Increased \\
\hline De C et al. [26] & Multicenter study- 3 sites & Increased \\
\hline \hline
\end{tabular}


Covid-19, and Hip Fracture Mortality Rates in the Elderly at One Year: Impact and Implications

\begin{tabular}{|c|c|c|}
\hline Fadulelmola et al. [5] & Comparative study- 75 cases & Appears high \\
\hline Hall et al. [24] & Multicenter study 340 cases- 317 cases & Increased \\
\hline Dupley et al. [45] & Retrospective multi center review- 64 cases & Increased \\
\hline Kayani et al. [29] & Multicenter study- 340 cases & Increased \\
\hline LeBrun et al. [33] & Case control study- 59 cases & Increased \\
\hline Macey et al. [21] & Retrospective analysis - 76 cases & No increase \\
\hline Mackay et al. [47]g & Retrospective study- 214 cases & Increased \\
\hline Munos-Vivas et al. [22] & Retrospective multi center study- 136 cases & Increased \\
\hline Mahmood et al. [48] & Multicenter observational study-1633 cases & Increased \\
\hline Ojeda-Thiess et al. [49] & Retrospective study- 64 cases & Not increased \\
\hline Thakrar et al. [35] & Single center prospective study- 43 cases & Increased \\
\hline Orfanos et al. [50] & Retrospective case-control - 97 cases & Increased for men \\
\hline Wignall et al. [38] & Retrospective multi-center study 580 cases & Increased \\
\hline Wright et al. [52] & Retrospective study- 68 cases & Increased \\
\hline Narang et al. [17] & Prospective multicenter study- 10 sites & Increased \\
\hline Tripathy et al. [51] & Meta analysis & Increased \\
\hline Tyas et al. [46] & Retrospective analysis- 40,000 cases & Increased \\
\hline Wang et al. [37] & Systematic review & Increased \\
\hline
\end{tabular}

Note: * 30-day COVID-19 positive mortality rate was $14 \% ; 0 \%$ for non infected $[\mathrm{p}=.22]$

Key explanatory factors for the increase in 30-day mortality rates depicted above are:

- Staff redeployment issues

- Reorganization procedures

- Lack of continuity of ward based clinical care

- Possible fatigue and weakness due to COVID-19 disease $[53,54]$

Unfortunately, while efforts to treat hip fractures in the hospital setting can be carried out so that no excess COVID-19 impact on mortality emerges [49], efforts to avert hip fracture injuries among older community dwelling adults, which have been established for some time, including physical activity and pharmacologic strategies [48] may have been attenuated owing to the urgency of dealing with COVID-19 risk prevention.

\section{Discussion}

Hip fracture injuries, one of the most serious public health problems facing aging nations, remain immense challenges to health care systems worldwide, as well as the many older adults who, if they survive the injury, are frequently found to incur high rates of 30day post surgical morbidity, a validated hip fracture outcome measure [17], as well as possible recurrent hip fracture injuries. At the same time, even if data on 30-day mortality rates post hip fracture, are presently limited in scope, and do not include those who may have died after discharge home, the current COVID19 pandemic has clearly placed many older adults at risk for premature death or increased general morbidity, while those older adults already at high risk for hip fractures are likely to also be at high or substantive risk for COVID-19 [23]. This review, which sought to document the most current prospective and retrospective plus comparative studies examining 30day post-hip fracture surgery mortality data largely during the first wave of the COVID pandemic, showed what is published appears to demonstrate that while the rate of COVID-19 infections among admitted hip fracture cases is modest in most cases, there is an extremely high risk of an older hip fracture patient incurring excess 30 -day mortality rates in the presence of a positive COVID-19 test result.

While the causes of this devastating outcome other than the presence of infection that would explain this observed excess rate of post surgical mortality are unclear, given that comorbidity scores may not be an apparent factor in this regard [33], the fact that the pandemic has greatly impacted health service delivery, including the likelihood of those that are 
Covid-19, and Hip Fracture Mortality Rates in the Elderly at One Year: Impact and Implications

designed to help older community dwelling adults at risk for hip fractures maintain their optimal health, may be one explanation. Another may be linked to the significant drop in prescriptions of vitamin D and calcium, bone sparing medicines, and bone scan requests during the lockdown period [15]. Since the home remains the site where most fractures appear to presently occur [18], it is possible some older adults isolated in the community who sustain a fall and possible fracture, may experience unacceptably long lies thereafter because they could not 'get up' readily without help, and are weaker than desired, especially if they are COVID-19 positive. Most falls were found to occur from a standing height [18], thus factors such as dizziness along with weakness in the absence of home health care, as well hospital associated COVID19 transmission [5], cannot be excluded.

Indeed, since falls and hip fractures continue to occur at rates comparable to pre pandemic rates [60] and may be increasing, rather than decreasing [11], it appears that either service factors, or factors such as home safety or general health maintenance behaviors, such as medication intake, as well as nutritive intake, may be posing some additional unidentified cause for concern, especially among older frail community dwelling adults living in isolation. As per Sharivate and Kachooei [58], it is apparent thata possible relationship between COVID-19 infection and fragility hip fracture in elderly patients could be induced inadvertently as a result of excess fatigue and weakness due to COVID19 disease that should not be ignored.

This situation may be further compounded if basic care services needs are not clearly identified and provided, and especially in the event vaccines are not consistently effective in countering COVID-19 variant risk and severity. The data also do not include those hip fracture cases that died of respiratory insufficiency and multi organ failure before receiving surgery [59]. As well, the impact on recovery from surgery [61-65] is basically unknown, given that COVID-19 risk and severity [66] may still be a concern even among those previously COVID free, for example when they remain for excess periods of time in hospital to recover, or return home where they may still be at risk for COVID19 [49], pain, weakness, falls, and re-injury.

Thus, while not all groups concur, many show COVID-19 as being a potentially potent independent determinant of increased 30-day mortality rates for patients with a hip fracture [14, 81], especially among those older adults with multi morbidities and fragility diagnoses [47], even if surgery improves pulmonary ventilation, patient comfort, and overall patient stability [59]. Hence, efforts to minimize the risk of COVID-19 infections and transmission, plus continued vigilance to counter the risk of hip fractures of those older inactive, osteoporotic, and frail adults living alone in the community, whose management may be severely hampered by lockdowns [38], are clearly of particular salience in this regard [10, 67]. Current lessons learned in the past year, as well as those learned before the pandemic, when combined may be especially helpful in this regard, as may future efforts to study a broad, rather than a limited array of associated themes and topical research related issues[68].

By contrast, and bearing in mind the multi-factorial origin of hip fractures, and that those older adults with multiple comorbid health conditions, and who are now socially isolated may be more vulnerable than ever, and less active and frailer than pre COVID patients [67], an exaggerated excess burden of multiple negative costs and outcomes can be anticipated to emerge for some time to come in our view if no concerted immediate comprehensive effort to counter this situation is forthcoming, even if 60 day post discharge mortality rates are lower than $1 \%$ [83]. Indeed, even if hospital based transmissions are minimized [27], as discussed by Jain et al. [70], elderly patients with hip fractures associated with medical comorbidities such as diabetes, and hypertension are not only more likely to enter the hospital with COVID19 infections, but due to their reduced functional reserves and weakened immune systems, may take longer to recover, even if they survive when compared to older adults with no apparent health problem or coronavirus infection, which can impact patient care itself markedly and significantly [28]. The observed link between falls among older adults that are deemed to be associated in some way with COVID-19 infection, and appear to be the most common mechanism for hip fracture occurrences during the pandemic outbreak, along with the nature of the relationship between COVID-19 and osteoporotic fragility hip fracture in the elderly $[53,61]$, the high risk of hospital COVID-19 transmission and post operative length of stay [27], should be examined further $[71,73]$. 
Covid-19, and Hip Fracture Mortality Rates in the Elderly at One Year: Impact and Implications

Indeed, this appears imperative, because even if hip fracture rates among some elderly subgroups appear be declining [74], or similar to those of the year preceding the COVID pandemic [57, 77], and those who have the disease may not necessarily exhibit excess mortality rates [44], a high percentage of elderly cases who currently experience hip fracture injuries plus a COVID-19 infection, do appear to have a potentially worse hip fracture outcome than those who do not, regardless of location, and age [78]. Since hip fractures and related mortality rates are anticipated to increase in their own right due to fragility and poor bone health in the very near future [75], and while possible new waves of the corona virus and its variants are anticipated, along with current possible post hospital discharge infections [82], and persistent disruptions in operative and home care elder services $[69,78]$ it appears action is needed now more than ever to avert any further degree of preventable excess disability and personal devastation among the aging population and is strongly advocated.

To this end, and to counter the persistent rate of hip fracture injuries in the community, as well as the high associated post-surgery 30-day mortality rates for COVID-19 positive cases [82, 84, 85], along with possible persistent COVID-19 infection risk, obtaining a comprehensive picture of all aspects of the present issue, especially the role of averting complications and long term health negative outcomes of those current hip fracture surgical cases who survive, is clearly desirable. Until further data is forthcoming, the current body of research implies multi disciplinary and thoughtful timely patient focused bone health assessments, home safety interventions and education may help to markedly mitigate both hip fracture and COVID-19 risk more successfully than is presently observed, especially among highly vulnerable isolated older adult populations [5, 60, 76]. As well, very careful insightful hospital based approaches, practical tailored protocols for safe protective essential home care practices and personalized follow up care, along with periodic frailty assessments, may be especially helpful in preventing injurious falls, as well as subsequent hip fractures, and possible COVID-19 infection risk and increased frailty [84, 86], among vulnerable older adults, including those returning home post surgery. In this regard, those with multiple comorbid health conditions, those who smoke, those with diabetes, sarcopenia, impaired cognition, and depression, among other age associated health risk correlates may need to be specifically assessed and duly targeted [29, 78-80, 84-86] as regards:
- Medication and environmental safety
- Prevention of influenza type infections
- Unsafe behaviours, including excess alcohol use
- Exercise practices
- Importance of adequate sleep
- Importance of adherence to health recommendations
- Possible vitamin C, D and calcium supplementation

\section{CONCLUSION}

Hip fractures among the elderly continue to result in excess health care as well as immense personal costs to society.

To avert the debilitating costly outcomes of currently hospitalized hip fracture cases, especially those with a parallel COVID-19 diagnosis, more timely upstream preventive efforts against both COVID-19 as well as hip fractures among at risk elders living in the community are strongly indicated.

To impact the immense current burden of hip fracture injuries and their generally adverse outcomes, future work to carefully examine the key risk factors that currently influence excess hip fracture incidence and mortality rates among vulnerable older adults, as well as the most promising modes of mitigation is strongly indicated as well.

\section{REFERENCES}

[1] Stevens JA, Rudd RA. The impact of decreasing U.S. hip fracture rates on future hip fracture estimates. Osteoporos Int. 2013;24(10):27252728. doi: $10.1007 /$ s00198-013-2375-9.

[2] Unim B, Minelli G, Da Cas R, Manno V, Trotta F, Palmieri L, et al. Trends in hip and distal 
Covid-19, and Hip Fracture Mortality Rates in the Elderly at One Year: Impact and Implications

femoral fracture rates in Italy from 2007 to 2017. Bone. 2021;142:115752. doi: 10.1016/j. bone.2020.115752.

[3] Leal J, Gray AM, Prieto-Alhambra D, Arden NK, Cooper C., et al. Impact of hip fracture on hospital care costs: a population-based study. Osteoporos Int. 2016:27(2),549-558. doi:10.1007/s00198015-3277-9

[4] Orces CH, Gavilanez EL. Increasing hip fracture rates among older adults in Ecuador: analysis of the National Hospital Discharge System, 19992016. Arch Osteoporos. 2017;12(1):109. doi: 10.1007/s11657-017-0410-8.

[5] Fadulelmola A, Gregory R, Gordon G., Smith F, JenningsA.TheimpactofCOVID-19infection onhip fractures 30-day mortality. Trauma Aug 27, 2020. https://doi.org/10.1177/1460408620951352

[6] Wang ZC, Jiang W, Chen X, Yang L, Wang H, Liu YH. Systemic immune-inflammation index independently predicts poor survival of older adults with hip fracture: a prospective cohort study. BMC Geriatr. 2021;21(1):155. doi: 10.1186/s12877-021-02102-3.

[7] Tran O, Silverman S, Xu X, Bonafede M, Fox $\mathrm{K}, \mathrm{McDermott} \mathrm{M}$, et al. Long-term direct and indirect economic burden associated with osteoporotic fracture in US postmenopausal women. Osteoporos Int. 2021 Jan 7. doi: 10.1007/ s00198-020-05769-3.

[8] Gong XF, Li XP, Zhang LX, Center JR, Bliuc D, Shi $\mathrm{Y}$, et al. Current status and distribution of hip fractures among older adults in China. Osteoporos Int. 2021 Mar 2. doi: 10.1007/s00198-02105849-y..

[9] Lin KB, Yang NP, Lee YH, Chan $\mathrm{CL}$, Wu CH, et al. The incidence and factors of hip fractures and subsequent morbidity in Taiwan: an 11year population-based cohort study. PLoS One. 2018;13(2):e0192388.

[10] Wilson RT. Wallace RB. Trends in hip fracture incidence in young and older adults. Am J Public Hlth. 2007;97:1734-1735.

[11] Carkci E, Polat B, Polat A, Peker B, Öztürkmen Y. The effect of the Coronavirus 2019 (COVID-19) pandemic on the number and characteristics of orthopedic trauma patients in a tertiary care hospital in Istanbul. Cureus. 2021;13(1):e12569. doi: $10.7759 /$ cureus.12569.

[12] Bhat AK, Kamath KS. Comparative study of orthopaedic trauma pattern in COVID lockdown versus non-covid period in a tertiary care centre. J Orthop. 2021;23:1-7. doi: 10.1016/j. jor.2020.11.008.

[13] Dolci A, Marongiu G, Leinardi L, Lombardo M, Dessì G, Capone A. The epidemiology of fractures and muskulo-skeletal traumas during COVID-19 lockdown: a detailed survey of 17.591 patients in a wide Italian Metropolitan Area. Geriatr Orthop Surg Rehabil. 2020;11:2151459320972673. doi: $10.1177 / 2151459320972673$.

[14] Abeygunasekara T, Lekamwasam S, Lenora J, Alwis G. Current incidence and future projections of fragility hip fractures in Sri Lanka. Arch Osteoporos. 2020:178. doi: 10.1007/s11657020-00848-8.

[15] Stephens A, RuddH,Stephens E, Ward J.Secondary prevention of hip fragility fractures during the COVID-19 pandemic: service evaluation of "MRS BAD BONES". JMIR Aging. 2020;3(2):e25607. doi: $10.2196 / 25607$.

[16] Narula S, Lawless A, D’Alessandro P, Jones CW, Yates P, Seymour H. Clinical Frailty Scale is a good predictor of mortality after proximal femur fracture: a cohort study of 30-day and one-year mortality. Bone Jt Open. 2020;1(8):443-449. doi: 10.1302/2633-1462.18.BJO-2020-0089.R1..

[17] Narang A, Chan G, Aframian A, Ali Z, Carr A, Goodier $\mathrm{H}$, et al. Thirty-day mortality following surgical management of hip fractures during the COVID-19 pandemic: findings from a prospective multi-centre UK study. Int Orthop. 2021;45(1):2331. doi: 10.1007/s00264-020-04739-y.

[18] Zhu Y, Chen W, Xin X, Yin Y, Hu J, Lv H, et al. Epidemiologic characteristics of traumatic fractures in elderly patients during the outbreak of coronavirus disease 2019 in China. Int Orthop. 2020;44(8):1565-1570. doi: 10.1007/s00264020-04575-0.

[19] Jürisson M, Raag M, Kallikorm R, Lember M, Uusküla, A. The impact of hip fracture on mortality in Estonia: a retrospective populationbased cohort study. BMC Musculoskelet Disord. 2017;18(1):243. 
Covid-19, and Hip Fracture Mortality Rates in the Elderly at One Year: Impact and Implications

[20] Rabanal LLevot JM, Moreno Suarez FM, Merodio Gómez A, Solar Herrera A, Tejón Pérez G. Hip fracture in elderly and COVID-19 infection. Report of 3 cases. Rev Esp Anestesiol Reanim. 2021;68(2):99-102. doi: 10.1016/j. redar.2020.05.015.

[21] Macey ARM, Butler J, Martin SC, Tan TY, Leach WJ, Jamal B. 30-day outcomes in hip fracture patients during the COVID-19 pandemic compared to the preceding year. Bone Jt Open. 2020;1(7):415419. doi: $10.1302 / 2633-1462.17$.

[22] Muñoz Vives JM, Jornet-Gibert M, CámaraCabrera J, Esteban PL, Brunet L, Delgado-Flores $\mathrm{L}$, et al; Spanish HIP-COVID Investigation Group. Mortality rates of patients with proximal femoral fracture in a worldwide pandemic: preliminary results of the Spanish HIP-COVID Observational Study. J Bone Joint Surg Am. 2020;102(13):e69. doi: 10.2106/JBJS.20.00686.

[23] Biarnés-Suñé A, Solà-Enríquez B, González Posada MÁ, Teixidor-Serra J, García-Sánchez Y, Manrique Muñóz S. Impact of the COVID19 pandemic on the mortality of the elderly patient with a hip fracture. Rev Esp Anestesiol Reanim. 2021;68(2):65-72. English, Spanish. doi: 10.1016/j.redar.2020.10.003.

[24] Hall AJ, Clement ND, Farrow L, MacLullich AMJ, Dall GF, Scott CEH, et al; IMPACT-Scot Study Group. IMPACT-Scot report on COVID-19 and hip fractures. Bone Joint J. 2020;102-B(9):12191228. doi: 10.1302/0301-620X.102B9.BJJ-20201100.R1.

[25] Arafa M, Nesar S, Abu-Jabeh H, Jayme MOR, Kalairajah Y. COVID-19 pandemic and hip fractures: impact and lessons learned. Bone Jt Open. 2020;1(9):530-540. doi: 10.1302/26331462.19.BJO-2020-0116.R1.

[26] De C, Wignall A, Giannoudis V, Jimenez A, Sturdee $\mathrm{S}$, Aderinto J, et al. Peri-operative Outcomes and predictors of mortality in COVID-19 positive patients with hip fractures: a multicentre study in the UK...Indian J Orthop. 2020;54(Suppl 2):111. doi: $10.1007 / s 43465-020-00272-7$.

[27] Ward AE, Tadross D, Wells F, Majkowski L, Naveed U, Jeyapalan R, et al. The impact of COVID-19 on morbidity and mortality in neck of femur fracture patients: a prospective case-control cohort study. Bone Jt Open. 2020;1(11):669-675. doi: 10.1302/2633-1462.111.BJO-2020-0141.R1.

[28] Egol KA, Konda SR, Bird ML, Dedhia N, Landes EK, Ranson RA, et al; NYU COVID Hip Fracture Research Group. Increased mortality and major complications in hip fracture care during the COVID-19 pandemic: a New York City perspective. J Orthop Trauma. 2020;34(8):395-402. doi: 10.1097/BOT.0000000000001845.

[29] Kayani B, Onochie E, Patil V, Begum F, Cuthbert $\mathrm{R}$, Ferguson D, et al. The effects of COVID19 on perioperative morbidity and mortality in patients with hip fractures. Bone Joint J. 2020;102-B(9):1136-1145. doi: 10.1302/0301620X.102B9.BJJ-2020-1127.R1.

[30] Al Farii H, Al Rawahi S, Samaila E, Lavini F, Magnan B, Al Maskari S. Thirty-day mortality in COVID-19 positive patients with hip fractures: a case-series and literature review. Geriatr Orthop Surg Rehabil. 2020;11:2151459320972681. doi: $10.1177 / 2151459320972681$.

[31] Kumar P, Jindal K, Aggarwal S, Kumar V, Rajnish RK. 30-Day mortality rate in hip fractures among the elderly with coexistent COVID-19 infection: a systematic review. Indian J Orthop. 2021:1-11. doi: 10.1007/s43465-021-00386-6.

[32] Crozier-Shaw G, Hughes AJ, Conlon B, Sheehan E, Merghani K. Hip fracture care during Covid-19: a regional trauma centre's experience. Ir J Med Sci. 2021;3:1-6. doi: 10.1007/s11845-020-02476-0.

[33] LeBrun DG, Konnaris MA, Ghahramani GC, Premkumar A, DeFrancesco CJ, Gruskay JA, et al. Hip fracture outcomes during the COVID-19 pandemic: early results from New York. J Orthop Trauma. 2020;34(8):403-410. doi: 10.1097/ BОT.0000000000001849.

[34] Cheung ZB, Forsh DA. Early outcomes after hip fracture surgery in COVID-19 patients in New York City. J Orthop. 2020:21:291-296. doi: 10.1016/j.jor.2020.06.003.

[35] Thakrar A, Chui K, Kapoor A, Hambidge, J. 30day mortality rate of patients with hip fractures during the COVID-19 pandemic: a single centre prospective study in the United Kingdom. J Orthop Trauma. 2020;34(9):e325-e329. doi: 10.1097/BOT.0000000000001889. 
Covid-19, and Hip Fracture Mortality Rates in the Elderly at One Year: Impact and Implications

[36] Rasidovic D, Ahmed I, Thomas C, Kimani PK, Wall P, Mangat K; NOF-COV19 Study Collaborative Group. Impact of COVID-19 on clinical outcomes for patients with fractured hip: a multicentre observational cohort study. Bone Jt Open. 2020;1(11):697-705. doi: 10.1302/26331462.111.BJO-2020-0132.R1..

[37] Wang KC, Xiao R, Cheung ZB, Barbera JP, Forsh DA. Early mortality after hip fracture surgery in COVID-19 patients: a systematic review and meta-analysis. J Orthop. 2020;22:584-591. doi: 10.1016/j.jor.2020.11.012.

[38] Wignall A, Giannoudis V, De C, Jimenez A, Sturdee S, Nisar S, et al. The impact of COVID-19 on the management and outcomes of patients with proximal femoral fractures: a multi-centre study of 580 patients. J Orthop Surg Res. 2021;16(1):155. doi: 10.1186/s13018-021-02301-z..

[39] Lim MA, Pranata R. Coronavirus disease 2019 (COVID-19) markedly increased mortality in patients with hip fracture - a systematic review and meta-analysis. J Clin Orthop Trauma. 2021;12(1):187-193. doi: 10.1016/j. jcot.2020.09.015.

[40] Clement ND, Hall AJ, Makaram NS, Robinson PG, Patton RFL, Moran M, et al. MPACT-Restart: the influence of COVID-19 on postoperative mortality and risk factors associated with SARS-CoV-2 infection after orthopaedic and trauma surgery. Bone Joint J. 2020;102-B(12):1774-1781. doi: 10.1302/0301-620X.102B12.BJJ-2020-1395.R2.

[41] Serra-Torres M, Barreda R, Weaver D, TorresReveron A. Delayed presentation of patients with hip fractures during the COVID-19 "Stayat-Home" order in the southmost region of the United States. Adv Orthop. 2021;2021:8822004. doi: $10.1155 / 2021 / 8822004$.

[42] Ishii K, Kurozumi T, Suzuki T, Matsui K, Inui T, Nakayama Y, et al. Impact of the COVID-19 pandemic on a trauma center of a university hospital in Japan. J Orthop Sci. 2020 Dec 29:S0949-2658(20)30360-2. doi: 10.1016/j. jos.2020.11.018.

[43] Burahee AS, Barry VE, Sutcliffe RP, Mahroof S. Older patients with proximal femur fractures and SARS-CoV-2 infection - an observational study. SICOT J. 2021;7:5. doi: 10.1051/sicotj/2021001.
[44] Dupley L, Oputa TJ, Bourne JT; North West COVID NOF Study Group. 30-day mortality for fractured neck of femur patients with concurrent COVID19 infection. Eur J Orthop Surg Traumatol. 2021;31(2):341-347. doi: 10.1007/s00590-02002778-0.

[45] Tyas B, Wilkinson M, Singisetti K. Effect of Covid-19 on best practice care of hip fracture patients: an analysis from the National Hip Fracture Database (NHFD). Surgeon. 2021:S1479-666X(21)00024-X. doi: 10.1016/j. surge.2021.01.003.

[46] Mackay ND, Wilding CP, Langley CR, Young J. The impact of COVID-19 on trauma and orthopaedic patients requiring surgery during the peak of the pandemic: a retrospective cohort study. Bone Jt Open. 2020;1(9):520-529. doi: 10.1302/26331462.19.BJO-2020-0108.R1.

[47] Mahmood A, Rashid F, Limb R, Cash T, Nagy MT, Zreik N, et al. Coronavirus infection in hip fractures (CHIP) study. Bone Joint J. 2021:1-6. doi: 10.1302/0301-620X.103B.BJJ-2020-1862. R1.

[48] Ojeda-Thies C, Cuarental-García J, García-Gómez E, Salazar-Zamorano CH, Alberti-Maroño J, Ramos-Pascua LR. Hip fracture care and mortality among patients treated in dedicated COVID-19 and non-COVID-19 circuits. Eur Geriatr Med. 2021:1-9. doi: 10.1007/s41999-021-00455-x. PMC7867866.

[49] Orfanos G, Al Kaisi K, Jaiswal A, Lim J, Youssef B. The effect of COVID-19 pandemic on the care of fragility hip fracture patients in the United Kingdom. a case control study in a major trauma centre. Surgeon. 2021:S1479-666X(21)00029-9. doi: 10.1016/j.surge.2021.01.008.

[50] Tripathy SK, Varghese P, Panigrahi S, Panda BB, Velagada S, Sahoo SS, et al. Thirty-day mortality of patients with hip fracture during COVID19 pandemic and pre-pandemic periods: A systematic review and meta-analysis. World J Orthop. 2021;12(1):35-50. doi: 10.5312/wjo. v12.i1.35.

[51] Wright EV, Musbahi O, Singh A, Somashekar $\mathrm{N}$, Huber $\mathrm{CP}$, Wiik AV. Increased perioperative mortality for femoral neck fractures in patients 
Covid-19, and Hip Fracture Mortality Rates in the Elderly at One Year: Impact and Implications

with coronavirus disease 2019 (COVID-19): experience from the United Kingdom during the first wave of the pandemic. Patient Saf Surg. 2021;15(1):8. doi: 10.1186/s13037-02000279-x.

[52] Hadfield JN, Gray AC. The evolving COVID-19 effect on hip fracture patients. Injury. 2020;51(7):14111412. doi: 10.1016/j.injury.2020.06.006.

[53] Upadhyaya GK, Iyengar K, Jain VK, Vaishya R. Challenges and strategies in management of osteoporosis and fragility fracture care during COVID-19 pandemic. J Orthop. 2020:21:287290.

[54] Minarro JC, Zamorano-Moyano C, UrbanoLuque MT, Arenas-de Larriva AP, IzquierdoFernández A, Quevedo-Reinoso R. Is COVID19 affecting the incidence of hip fractures? Injury, 2020;51(10):2329. doi:10.1016/j. injury.2020.07.018

[55] Donovan RL, Tilston T, Frostick R, Chesser T. Outcomes of orthopaedic trauma services at a UK major trauma centre during a national lockdown and pandemic: the need for continuing the provision of services. Cureus. 2020;12(10):e11056. doi: 10.7759/ cureus.11056.

[56] Ogliari G, Lunt E, Ong T, Marshall L, Sahota O. The impact of lockdown during the COVID-19 pandemic on osteoporotic fragility fractures: an observational study. Arch Osteoporos. 2020;15(1):156. doi: 10.1007/s11657-02000825-1.

[57] Shariyate MJ, Kachooei AR. Association of new coronavirus disease with fragility hip and lower limb fractures in elderly patients. Arch Bone Jt Surg. 2020;8(S1):297-301. doi: 10.22038/ abjs.2020.47626.2333.

[58] Catellani F, Coscione A, D'Ambrosi R, Usai L, Roscitano C, Fiorentino G. Treatment of proximal femoral fragility fractures in patients with COVID-19 D $\backslash$ during the SARS-CoV-2 outbreak in Northern Italy. J Bone Joint Surg Am. 2020;102(12):e58. doi: 10.2106/JBJS.20.00617.

[59] Ribau A, Vale J, Xará-Leite F, Rodrigues-Pinto R. Impact of COVID-19 pandemic and national lockdown in an orthopaedic and traumatology department-a comparison with the homologous period of 2019. Porto Biomed J. 2021;6(1):e109. doi: $10.1097 /$ j.pbj.0000000000000109.

[60] Oakes B, Bolia IK, Weber AE, Petrigliano FA. Vitamin $\mathrm{C}$ in orthopedic practices: Current concepts, novel ideas, and future perspectives. J Orthop Res. 2020 Dec 10. doi: 10.1002/ jor.24947.

[61] Brzezińska 0, Łukasik Z, Makowska J, Walczak K. Role of vitamin $\mathrm{C}$ in osteoporosis development and treatment-a literature review. Nutrients. 2020;12(8):2394. doi: 10.3390/nu12082394.

[62] Sun Y, Liu C, Bo Y, You J, Zhu Y, Duan D, Cui H, $\mathrm{Lu}$ Q. Dietary vitamin $\mathrm{C}$ intake and the risk of hip fracture: a dose-response meta-analysis. Osteoporos Int. 2018;29(1):79-87. doi: 10.1007/ s00198-017-4284-9.

[63] Malmir H, Shab-Bidar S, Djafarian K. Vitamin C intake in relation to bone mineral density and risk of hip fracture and osteoporosis: a systematic review and meta-analysis of observational studies. Br J Nutr. 2018;119(8):847-858. doi: 10.1017/S0007114518000430.

[64] Torbergsen AC, Watne LO, Wyller TB, Frihagen F, Strømsøe K, Bøhmer T, Mowe M. Micronutrients and the risk of hip fracture: case-control study. Clin Nutr. 2017;36(2):438-443. doi: 10.1016/j. clnu.2015.12.014.

[65] Iddir M, Brito A, Dingeo G, Fernandez Del Campo SS, Samouda H, La Frano MR, et al. Strengthening the immune system and reducing inflammation and oxidative stress through diet and nutrition: considerations during the COVID-19 crisis. Nutrients. 2020;12(6):1562. doi: 10.3390/ nu12061562.

[66] Slullitel PA, Lucero CM, Soruco ML, Barla JD, Benchimol JA, Boietti BR, et al; HipFEIR [Hip Fracture in the Elderly - Institutional Register] Study Group. Prolonged social lockdown during COVID-19 pandemic and hip fracture epidemiology. Int Orthop. 2020 Oct;44(10):18871895. doi: 10.1007/s00264-020-04769-6.

[67] Peng G, Guan Z, Hou Y, Gao J, Rao W, Yuan X, et al. Depicting developing trend and core knowledge of hip fracture research: a bibliometric and visualised analysis. J Orthop Surg Res. 2021;16(1):174. doi: 10.1186/s13018-02102292-x. 
Covid-19, and Hip Fracture Mortality Rates in the Elderly at One Year: Impact and Implications

[68] Sheikh Saleem J, Ali Fazal M. Fragility hip fractures in the COVID-19 pandemic: a local experience in the United Kingdom. Int J Orthop Trauma Nurs. 2020 Aug 25:100817. doi: 10.1016/j. ijotn.2020.100817.

[69] Jain VK, Lai H, Patralekh MK.Vaishya R. Fracture management during COVID_19 pandemic: a systematic review. J Clin Orthop Trauma. 2020;11(4):S431-S441.

[70] Gawronska K, Lorkowski J. Falls as one of the atypical presentations of COVID-19 in older population. Geriatr Orthop Surg Rehabil. 2021 Feb 21;12:2151459321996619. doi: $10.1177 / 2151459321996619$.

[71] Groenendijk I, Kramer CS, den Boeft LM, Hobbelen HSM, van der Putten GJ, de Groot LCPGM. Hip fracture patients in geriatric rehabilitation show poor nutritional status, dietary intake and muscle health. Nutrients. 2020;12(9):2528. doi: 10.3390/nu12092528.

[72] González-Martín D, Álvarez-De la Cruz J, MartínVélez P, Boluda-Mengod J, Pais-Brito JL, HerreraPérez M. Quantitative and qualitative analysis of the influence of confinement by COVID-19 in fracture patients entered in a traumatology service at a third level hospital. Rev Esp Cir Ortop Traumatol. 2020:S1888-4415(20)30169-7.

[73] Pluskiewicz W, Wilk R, Adamczyk P, Hajzyk M, Swoboda M, Sladek A, Koczy B. The incidence of arm, forearm, and hip osteoporotic fractures during early stage of COVID-19 pandemic. Osteoporos Int. 2021 Jan 30:1-5. doi: 10.1007/ s00198-020-05811-4.

[74] Napoli N, Elderkin AL, Kiel DP, Khosla S. Managing fragility fractures during the COVID19 pandemic. Nat Rev Endocrinol. 2020:1-2. doi:10.1038/s41574-020-0379-z

[75] Leal JA, Garcia LF, Peña OR, Gomez-Gelvez A. Patients aged ninety years and older are exposed to increased risk of one-year mortality after hip fractures. Eur J Orthop Surg Traumatol. 2021:Mar 2. doi: 10.1007/s00590-021-02918-0.

[76] Greenhalgh M, Dupley L, Unsworth R, Boden
R. Where did all the trauma go? A rapid review of the demands on orthopaedic services at a UK Major Trauma Centre during the COVID-19 pandemic. Int J Clin Pract. 2020 Aug 27:e13690. doi: 10.1111/ijcp.13690.

[77] Lim MA, Kurniawan AA. Dreadful consequences of sarcopenia and osteoporosis due to COVID19 containment. Geriatr Orthop Surg Rehabil. 2021 Feb 10;12:2151459321992746. doi: $10.1177 / 2151459321992746$.

[78] BhattacharyyaT.Osteoporoticfracturesinthetime of COVID-19. J Bone Miner Res. 2020;10.1002/ jbmr.4113. doi:10.1002/jbmr.4113

[79] Bartosch P, Malmgren L, Kristensson J, McGuigan $\mathrm{FE}$, Akesson KE. In community-dwelling women frailty is associated with imminent risk of osteoporotic fractures. Osteoporos Int. $2021 \mathrm{Mar}$ 4. doi: 10.1007/s00198-021-05886-7.

[80] Fessler J, Jacobsen T, Lauritzen JB, Jørgensen HL. Mortality among hip fracture patients infected with COVID-19 perioperatively. Eur J Trauma Emerg Surg. 2021 Mar 11. doi: 10.1007/s00068021-01634-y.

[81] Greensmith TSW, Faulkner AC, Davies PSE, Sinnerton RJH, Cherry JV, Supparamaniam S, MacInnes A, Dalgleish S. Hip fracture care during the 2020 COVID-19 first-wave: a review of the outcomes of hip fracture patients at a Scottish Major Trauma Centre. Surgeon. 2021 Feb 25:S1479-666X(21)00033-0. doi: 10.1016/j. surge.2021.01.012.

[82] Wickramarachchi L, Peters J, Thakrar A, Wong JM, Mazis G, Beckles V, Lacey S, Chin K. A dualsite trauma system during COVID-19 pandemic - our experience in a high-risk area with 60-day mortality report. J Orthop. 2021;24:42-46. doi: 10.1016/j.jor.2021.02.013.

[83] Craig J, Maguire M, Shevlin SP, Black ND. The effects of COVID-19 on hip fracture management and mortality in a regional trauma centre. Anaesthesia. 2020;10.1111/anae.15323.

[84] Haskel JD, Lin CC, Kaplan DJ, Dankert JF, Merkow D, Crespo A, etal. Hip fracturevolumedoesnotchange

Open Journal of Geriatrics V4 . I1 . 2021 
Covid-19, and Hip Fracture Mortality Rates in the Elderly at One Year: Impact and Implications

at a New York City Level 1 Trauma Center during frailty is associated with imminent risk of a period of social distancing. Geriatr Orthop Surg osteoporotic fractures. Osteoporos Int. $2021 \mathrm{Mar}$ Rehabil. 2020 Nov 11;11:2151459320972674. 4. doi: 10.1007/s00198-021-05886-7. doi: $10.1177 / 2151459320972674$.

[85] Bartosch P, Malmgren L, Kristensson J, McGuigan FE, Akesson KE. In community- dwelling women

Citation: Ray Marks. Covid-19, and Hip Fracture Mortality Rates in the Elderly at One Year: Impact and Implications. Open Journal of Geriatrics. 2021; 4(1): 01-13. DOI: https://doi.org/10.22259/2639359X.0401001

Copyright: (C) 2021 Ray Marks. This is an open access article distributed under the Creative Commons Attribution License, which permits unrestricted use, distribution, and reproduction in any medium, provided the original work is properly cited. 